Article

\title{
In Silico Mapping of Essential Residues in the Catalytic Domain of PDE5 Responsible for Stabilization of Its Commercial Inhibitors
}

\author{
Ivan Pires de Oliveira ${ }^{1, * \mathbb{D}}$, Caroline Honaiser Lescano ${ }^{2}$ and Gilberto De Nucci ${ }^{1,2}$ \\ 1 Department of Pharmacology, Institute of Biomedical Sciences, University of São Paulo, \\ 05508-900 São Paulo-SP, Brazil; denuccigilberto@gmail.com \\ 2 Department of Pharmacology, School of Medical Sciences, University of Campinas, 13083-887 Campinas-SP, \\ Brazil; carolinehonaiser@gmail.com \\ * Correspondence: ivan.pires.oliveira@gmail.com
}

Received: 9 September 2019; Accepted: 28 October 2019; Published: 1 November 2019

\begin{abstract}
Phosphodiesterase type 5 (PDE5) is an important enzyme associated with the hydrolysis of cyclic guanosine monophosphate (cGMP) to guanosine monophosphate (GMP). Due to the relevant role of second messenger cGMP as a mediator in many physiological processes, efforts have been converged to find a safe pharmacological approach, seeking a specific, selective and potent inhibitor of the PDE5 enzyme. There are five commercial drugs with potential for clinical use: tadalafil, sildenafil, avanafil, udenafil and vardenafil. Here, we applied molecular modeling to obtain different profiles of protein-ligand interactions by adopting distinct PDE5 structures, specifically PDBid:1XOZ and two extracted from molecular dynamics (MD) simulations. The results generated by molecular docking showed several possibilities for inhibitor interactions with the catalytic pocket. Tadalafil, sildenafil and vardenafil were clearly stabilized by Gln817 via a well-oriented hydrogen bond. Another set of different interactions, such as polar, hydrophobic, $\pi$-stacking, metal-ligand and electrostatic, were responsible for accommodating avanafil and udenafil. All of the ligands are discussed in detail with consideration of the distinct protein structures, and a profile of the probability of residue-ligand contact is suggested, with the most frequently observed being: Tyr612, His613, Ser661, Thr723, Asp724, Asp764, Leu765, Val782 and Phe786. The molecular interactions displayed herein confirm findings achieved by previous authors and also present new contacts. In addition, the discussion can help researchers obtain a molecular basis for planning new selective PDE5 inhibitors, as well as explain an inhibitor's experimental assays by considering the specific interactions occurring at the catalytic site.
\end{abstract}

Keywords: catalytic site; biocatalysis; cGMP; phosphodiesterase type 5; PDE5 inhibitors; molecular docking

\section{Introduction}

Phosphodiesterases (PDEs) are functionally distinct and highly regulated enzymes that have been classified into 11 families based on different genes in mammals [1]. These proteins are responsible for the hydrolysis of cyclic nucleotides and, among these enzymes, phosphodiesterase type 5 (PDE5) is considered particularly relevant for therapeutics [2]. PDE5 controls the level of intracellular cyclic guanosine monophosphate (cGMP) [3], which is an important mediator in many physiological processes including smooth muscle relaxation [3,4], secretion of ions [3-5], platelet function [6], natriuresis [7], and sperm motility [8], as well as processes such as cell growth and apoptosis [9]. Fundamental in intracellular signaling, cGMP is produced by soluble guanylate cyclase (sGC) or particulate guanylate cyclase (pGC) by the biocatalytic conversion of guanosine triphosphate (GTP) to cGMP; this event 
occurs in a broad spectrum of cells $[9,10]$. The level of cGMP in a cell is mainly determined by the balance between the activities of guanylate cyclase (synthesis) and phosphodiesterases that hydrolyze cGMP to guanosine monophosphate (GMP) [11]. The intracellular cGMP produced has its cellular functions exerted by its binding to specific molecular targets, such as kinase proteins, ion channels, and phosphodiesterases [12]. These enzymes, especially PDE5, modulate the level of this second messenger and they are present in many tissues, including the corpus cavernosum [13], platelets [14], and vascular smooth muscle cells [14,15].

Inhibitors of PDE5 have gained prominence in the scientific field with advances in understanding the physiological functions of the PDE5 enzyme [16-18]. Specifically, such inhibitors were originally synthesized for treatment of hypertension, pulmonary hypertension, coronary heart disease and angina [19]. However, later inhibitors emerged as an interesting indication for erectile dysfunction, because the expression of PDE5 is more abundant in the human corpus cavernosum compared to other tissues. This is an important factor because it is considered to be the main reason for the clinical efficacy of these inhibitors [13,19]. All PDE5 inhibitors share similarities and they compete for active site binding with cGMP. In general, they act to inhibit PDE5 by blocking cGMP binding at the catalytic site, thus preventing the hydrolysis of cGMP to guanosine monophosphate [20,21]. Currently, sildenafil, tadalafil and vardenafil are the most common drugs for the treatment of erectile dysfunction approved by the Food and Drug Administration (FDA) [22]. Despite the drugs' efficiency, some PDE5 inhibitors have collateral effects which include headache and nasal congestion. The effects are due to the dilation of arterial vessels, as well as non-selective inhibition of PDE1 which also hydrolyzes cGMP and is located predominantly in vascular smooth muscle cells [19,22]. In addition, PDE5 inhibitors are not only correlated with erectile dysfunction therapy; for example, tadalafil has recently been approved for the treatment of benign prostatic hyperplasia, pulmonary hypertension and lower urinary tract symptoms [22-24], and recent studies have pointed to PDE5 inhibitors as a new strategy for the treatment of certain types of tumors [25], demonstrating the importance of these compounds [26]. Researchers have investigated the correlation of important residues in PDE5 with the selectivity of these ligands [27], which directly impacts the therapeutic applications of the aforementioned drugs. It has been reported that overexpression of PDE5 occurs in many carcinomas, including prostate [28], mamma [29], and colon (adenocarcinomas), as well as in cancer in lung cells and chronic lymphocytic leukemia cells $[25,29]$. In this sense, the use of PDE5 inhibitors has motivated work that investigates the chemopreventive mechanisms of such agents [25]. This is a strong motivator to better understand the interactions of drugs with the enzyme catalytic pocket and consequently to help in the development of novel PDE5 inhibitors with greater selectivity for novel therapeutic targets [30].

In silico analyses have been applied recently in order to understand the interactions of several phosphodiesterase types and different inhibitors in the catalytic pocket. These are powerful methods for predicting important residues and secondary structures which stabilize the inhibitors [31,32]. In this study, we were interested in understanding how the most common commercial PDE5 inhibitors interact with critical residues that comprise the catalytic domain of the target protein. To do this, we applied an in silico approach. The interactions were mapped using molecular docking, wherein different structures of the protein were adopted to get a better description of protein-ligand interactions and to eliminate correlations with the crystallographic structure. For this purpose, two independent molecular dynamics simulations of 80 ns were computed and the coordinates of the atoms used for docking studies, as described in detail in the methods. The five inhibitors selected for investigation in this work are widely discussed in the literature and have potential use in therapeutics.

\section{Materials and Methods}

\subsection{Protein Structure Details}

The enzyme PDE5 is a protein with a dimeric structure. It includes a C-terminal domain with a catalytic pocket of about 270 amino acids, which require two divalent metal ions for catalytic function 
$\left(\mathrm{Mg}^{2+}\right.$ and $\left.\mathrm{Zn}^{2+}\right)$ and are also present in other PDEs $[1,15,19,33]$. Moreover, the N-terminal domain presents the GAF-A and GAF-B regulatory subdomains, which are the allosteric binding regions for the cGMP enzymatic substrate, and also a phosphorylation site (at position Ser92) for kinase A and kinase $G$ proteins [34]. Details about the dimerization process and the PDE5 structure are discussed by Zoraghi et al. [35]. Interestingly, PDE5 is present in at least two different conformations, representing an inactive state and an active state, with widely different affinities for PDE5 inhibitors [16]. The transition between these conformations is triggered by several factors $[16,17]$ and is out of the scope of this paper, which consider only the active protein conformation.

The protein structure of human phosphodiesterase type 5 was chosen for molecular modeling studies from the crystallographic structure with a resolution of $1.37 \AA$ complexed with tadalafil, available from the Protein Data Bank (PDB) database (PDBid:1XOZ) [36] with a molecular mass of approximately $200 \mathrm{kDa}$. Besides the experimental structure, two independent molecular dynamics (MD) simulations of $80 \mathrm{~ns}$ were performed to avoid initial correlations with the tadalafil ligand. From the last step of the simulation trajectories, the coordinate file $(x, y, z)$ of the protein and cofactors $\left(\mathrm{Mg}^{2+}\right.$ and $\left.\mathrm{Zn}^{2+}\right)$ were generated for use in a molecular docking protocol; the result for each compound is presented herein as a sum of the three adopted independent structures.

\subsection{Molecular Dynamics Simulations Protocol}

Molecular dynamics simulations were performed without the tadalafil ligand. The initial configurations were constructed with Packmol [37], containing PDE5 centered in the simulation box with 27,000 water molecules and $\mathrm{Na}^{+}$and $\mathrm{Cl}^{-}$ions to neutralize the protein charges at concentrations close to $0.1 \mathrm{~mol} / \mathrm{L}$. The systems were simulated as follows: (i) with all atoms of the protein fixed, water molecules were relaxed by performing 1000 minimization steps by the gradient conjugate (GC) method, followed by 200 ps of MD simulation; (ii) keeping only the C $\alpha$ atoms of the protein fixed, 500 GC minimization steps were performed, followed by 200 ps of MD simulation; (iii) all PDE5 atoms were released with $2.2 \mathrm{~ns}$ of MD simulation; (iv) with the coordinates and final velocities of the previous step, the production run was started. For all systems, particle mesh Ewald (PME) was used, and periodic boundary conditions, applying a grid spacing value of $1.0 \AA$ and a cut-off of $12 \AA$, were adopted for non-bonded interactions. Two independent simulations of $80 \mathrm{~ns}$ were performed in an NPT ensemble at $1 \mathrm{~atm}$ and $310.15 \mathrm{~K}$, totaling $160 \mathrm{~ns}$ of simulation. Then, the last protein coordinates were adopted for molecular docking studies. The pressure was controlled in a Nosé-Hoover Langevin bath with a damping coefficient of $10 \mathrm{ps}^{-1}$. The CHARMM force field was used for PDE5 enzyme, ions, water and ligands [38]. The trajectories were computed using the NAMD program [39] and all of the visualizations and pictures were obtained with the VMD package [40].

\subsection{Molecular Docking Protocol}

The three-dimensional structures of the small molecules studied in this work were obtained from the PubChem database: avanafil $\left(\mathrm{C}_{23} \mathrm{H}_{26} \mathrm{ClN}_{7} \mathrm{O}_{3} ; 483.96 \mathrm{~g} / \mathrm{mol}\right.$; CID:9869929); sildenafil $\left(\mathrm{C}_{22} \mathrm{H}_{30} \mathrm{~N}_{6} \mathrm{O}_{4} \mathrm{~S} ; 474.58 \mathrm{~g} / \mathrm{mol}\right.$; CID:5212); tadalafil $\left(\mathrm{C}_{22} \mathrm{H}_{19} \mathrm{~N}_{3} \mathrm{O}_{4} ; 389.41 \mathrm{~g} / \mathrm{mol} ; \mathrm{CID}: 110635\right) ;$ udenafil $\left(\mathrm{C}_{25} \mathrm{H}_{36} \mathrm{~N}_{6} \mathrm{O}_{4} \mathrm{~S} ; 516.66 \mathrm{~g} / \mathrm{mol} \mathrm{CID}: 6918523\right)$ and vardenafil $\left(\mathrm{C}_{23} \mathrm{H}_{32} \mathrm{~N}_{6} \mathrm{O}_{4} \mathrm{~S} ; 488.61 \mathrm{~g} / \mathrm{mol} ; \mathrm{CID}: 110634\right)$ [41]. The three-dimensional structures of the compounds were minimized with the ChemBioDraw Ultra 12.0 program using the force field MMFF94 before undergoing the docking method (see Figure S1 in supplementary material). With the prepared PDE5 protein structures (crystallographic and from MD trajectories) and optimized compounds, the DockThor program was adopted for molecular docking calculations (online platform version https://dockthor.lncc.br/v2/\#). DockThor is a receptor-ligand docking server, which employs a multiple solution genetic algorithm to predict the best structures and their energies, considering a flexible ligand-rigid receptor model and the MMFF94S force field [42]. The genetic algorithm implemented in DockThor performs one million cycles (number of evaluations) to generate new individuals (structures) from randomly picked parents (initial population). The population size (750 structures) is constantly updated, according to the lowest energy structures 
generated, to the limit established by the number of evaluations, and the final set of structures is reserved. This process was performed independently 24 times ( 24 runs) and, at the end, the set of final structures was clustered using a root-mean-square deviation (RMSD) value of $1.0 \AA$ [42]. As suggested by the developers, the genetic algorithm values used were the defaults for optimized flexible molecules. The systems studied here were inserted into the platform as follows: first the protein coordinate file was loaded, followed by insertion of the file structures of the ligands and addition of the $\mathrm{Zn}^{2+}$ and $\mathrm{Mg}^{2+}$ cofactors; then, the complete protein was loaded onto the DockThor platform and submitted to a docking energy calculation. The docking grid coordinate center was defined by the spatial volume of the PDE5 catalytic site. Specifically, we used the center of the grid X: 8.0 Y: $-4.0 \mathrm{Z}:-8.0$, with $11 \AA$ of grid size on each axis.

\section{Results and Discussion}

\subsection{Catalytic Domain of PDE5 and Docking Validation}

It is known that the compounds discussed herein have high ability to interact with a specific region of the protein, the catalytic site, and prevent the hydrolysis of cGMP [20-22,36]. Therefore, we consider that the inhibitors act directly in the catalytic pocket of the PDE5 enzyme, as previously described by different research groups $[17,27,36,43]$, and not by an allosteric mechanism. According to previous studies, the PDE5 enzyme has a number of subdomains within the catalytic pocket: (i) the M site, a metal-binding site for ions and residues around $\mathrm{Mg}^{2+}$ and $\mathrm{Zn}^{2+}$; (ii) the Q pocket, a central region of the catalytic site; (iii) the H pocket, a hydrophobic pocket; and (iv) the L region, a subdomain associated with catalytic site opening, also named the lid region. Each of these regions exhibits particularities in their interaction with ligands, as detailed by the three-dimensional structure of the protein and the catalytic site in Figure 1A, which highlights some important amino acid residues: (i) M site (Asp764, His653, Asp654, His617, and ions $\mathrm{Zn}^{2+}$ and $\mathrm{Mg}^{2+}$ ); (ii) Q pocket (Phe820, His613, Tyr612, Ala767, Val782, Gln817 and Ile768); (iii) H pocket (Leu804, Phe786, Ala783, Ile813 and Phe787) and (iv) L region (Met816, Gly819, Phe664 and Ala823).

A

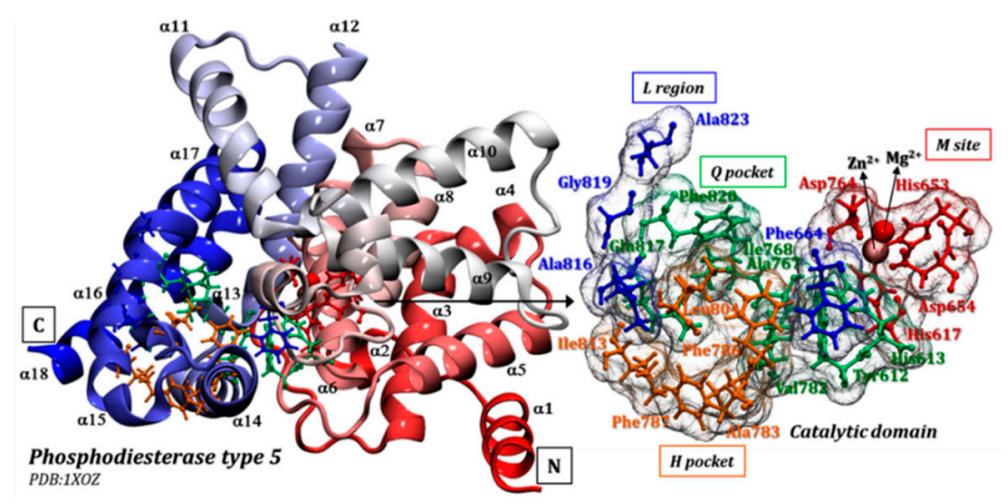

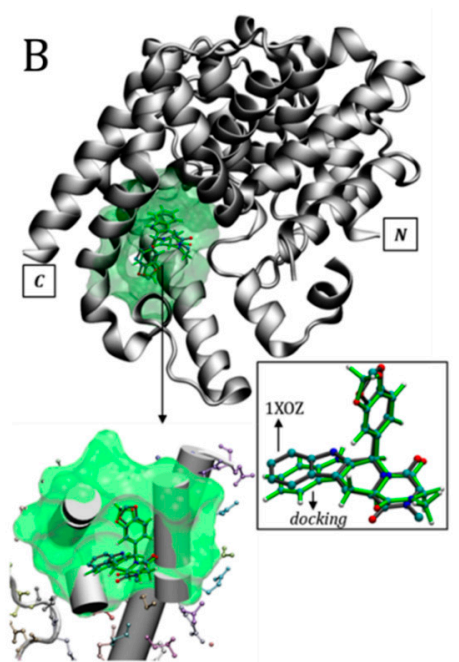

Figure 1. Details of the three-dimensional structure of the phosphodiesterase type 5 (PDE5) enzyme: (A) highlighting some amino acid residues that make up the catalytic domain and (B) comparative redocking of the conformational arrangements of the tadalafil inhibitor in the crystallographic structure (in grey) and using the molecular docking protocol (highlighted in green).

In general, it is possible to observe that the structures did not undergo any drastic conformational changes during the simulation trajectories (see supplementary material, Figure S2), and the structures obtained were practically overlapping, as shown by the RMSD values. However, it is possible to 
identify important mobilities of catalytic site residues, with conformational changes of side chain residues in all subdomains, for example: M site (His653, His617); H pocket (Ile813, Leu804); Q pocket (Gln817, His613) and L region (Met816). As highlighted above, residues from the catalytic domain are further away from the crystallographic structure and this should have an effect on the docking mode of the PDE5 inhibitors, as discussed below. It is worth noting that it is still a challenge to obtain representative protein structures for molecular docking that have no correlation with the original inhibitor widely discussed in the literature [44-47].

The molecular docking methodology was validated by the redocking method, using the tadalafil inhibitor and $1 \mathrm{XOZ}$ crystallographic structure. As shown in Figure 1B, overlap between ligand structures makes clear the ability of the program to predict the correct position of this inhibitor at the catalytic site of phosphodiesterase type 5; it was also recently validated for a significant set of enzymes and ligands [42]. In this sense, the genetic algorithm implemented in DockThor and the force field MMFF94S satisfied the needs of the system studied here.

\subsection{Tadalafil (Cialis $\left.{ }^{\circledR}\right)$}

Stabilization of the inhibitors in the catalytic domain can be understood from the different forms of protein-ligand interaction. Such intermolecular interactions can be observed by the "contact" between the inhibitory molecules with the different side chains or backbone of specific residues. Some of these specific interactions for tadalafil are detailed in Figure 2 (also shown in Figure S3), which shows a set of possibilities for protein-inhibitor interactions considering the different protein structures used in molecular docking. An important interaction known to stabilize the inhibitor tadalafil is the hydrogen bonding performed with Gln817 [17,27,48], as identified in the $1 \mathrm{XOZ}$ structure via redocking. Moreover, this glutamine was previously studied by our research group, wherein the quantitative importance of H-bonds to stabilize tadalafil was identified [49]. On the other hand, based on MD structures, different residues may also stabilize tadalafil. This may occur through polar interactions, which may be oriented sufficiently to form hydrogen bonds with residues Leu725 (backbone amine), Asn662, His613 and Tyr612, or there are possible hydrophobic interactions with Leu725, Leu804 and Phe786.

It is important to highlight the relevance of subtle changes in the protein structure which enable the sampling of several protein-ligand interactions (see Table S1 and Figure S2 in supplementary material for details). We found that the protein did not appear to have lost any active biological conformation as the RMSD values were relatively low, with no value above $2.0 \AA$. Thus, the analysis was within the active conformational space of the enzyme [50]. Another important aspect was identification of the magnesium ion as a stabilizing agent of the ligand in the structures obtained from MD simulations. It is known that $\mathrm{Mg}^{2+}$ and $\mathrm{Zn}^{2+}$ ions are important for stabilizing cGMP and also sildenafil, as shown by a previous study [27]. Here, we suggest that these ions can participate in the binding on the $M$ site by anchoring tadalafil. In addition, a set of specific residues contributed to tadalafil stabilization, including His613, Asp654, His657, Gly659, Val660, Ser661, Ans662, Thr723, Asp724, Leu725, Ala726, Leu765, Gln789 and Met805. 


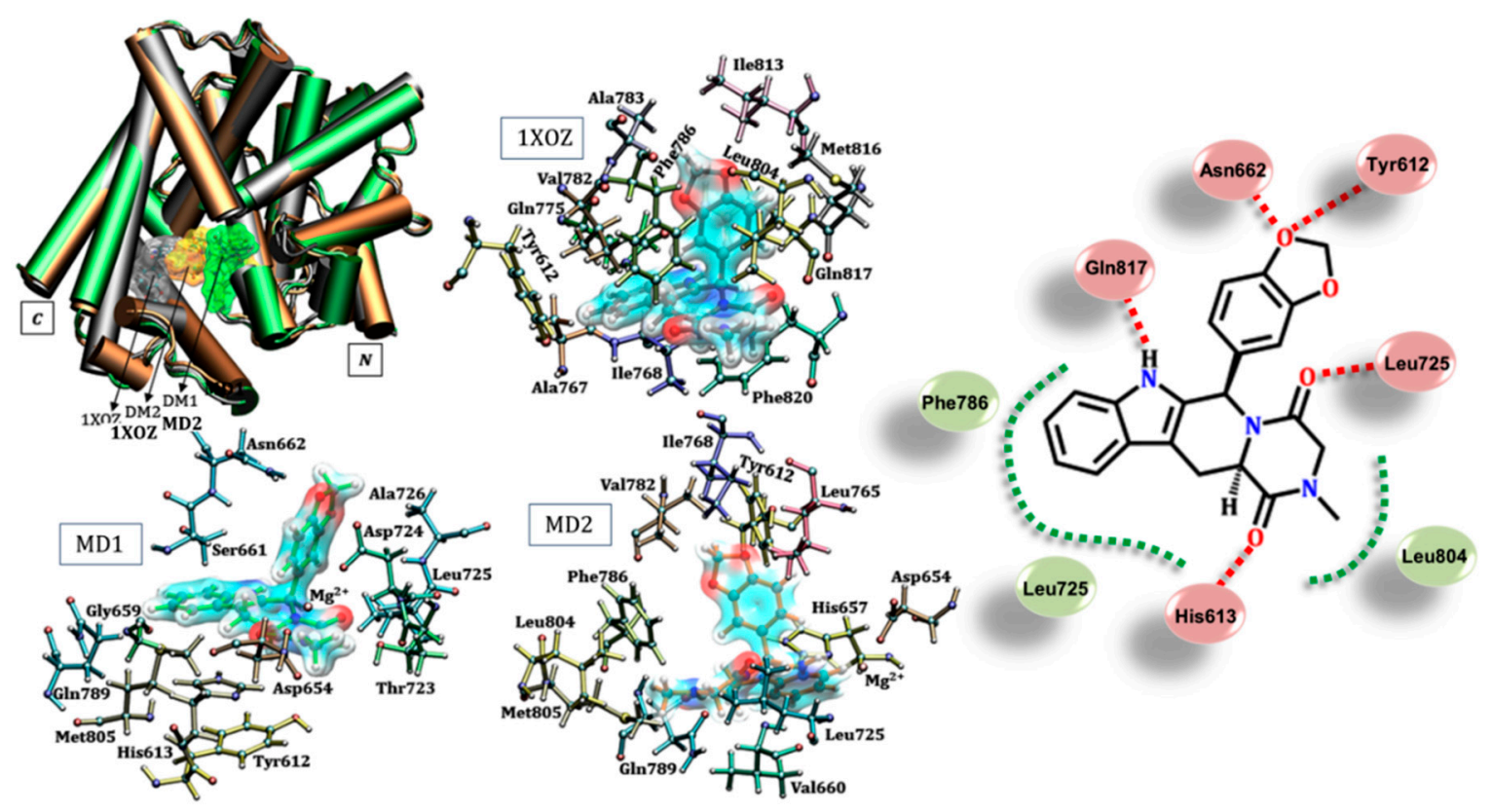

Figure 2. Interactions identified between the tadalafil and specific amino acids in the catalytic domain of PDE5 considering three different protein structures: crystallographic and two from 80 ns molecular dynamics (MD) simulations (MD1 and MD2). Residues considered only at distance equal to or less than $3.0 \AA$ from the ligand ( $\mathrm{r} \leq 3.0 \AA$ ) . Legend of interactions, red: hydrogen bond (and dipole-dipole); green: hydrophobic.

\subsection{Sildenafil (Viagra ${ }^{\circledR}$ )}

Sildenafil also forms a hydrogen bond with Gln817 in the 1XOZ structure by using a nitrogen of the pyrazole ring, which is different to the pyrimidine ring noted previously [27,51]. Moreover, another important contribution to the stabilization of sildenafil in the crystallographic structure may originate in the hydrophobic interactions with Leu765, Ala767, Ile768 and Phe820, as shown in Figure 3 (and Figure S4). An analysis of MD structures suggested other possibilities for accommodation of this inhibitor. These include hydrogen bonds between the imidazole ring of the side chain of His657 and His613 with the benzenesulfonamide group of the ligand, and interactions of the $\pi$-stacking type, which were observed between the benzene rings of Phe820 and sildenafil. Several important hydrophobic contributions may also be associated with other amino acid residues, such as Leu725, Val782 and Leu804, as depicted in Figure 3.

Unlike those observed for tadalafil, the positions obtained for the sildenafil inhibitor were centered on a specific region of the catalytic domain, as shown in Figure 3A. However, we were able to identify a change in the conformation of the ligand with specific interactions according to each protein structure used. An interesting aspect was the very close position assumed by sildenafil in the crystallographic structure and the structure extracted from the MD simulation, MD1. Therefore, despite the structural differences between these two structures (see Table S1 for RMSD values), a roughly equal set of residues stabilized sildenafil in the catalytic site: Tyr612, Ser661, Leu725, Asp764, Leu765, Val782, Phe786, Leu804 and Phe820. The interactions observed for the other MD structure showed an alternative way of accommodating the inhibitor within the catalytic pocket, which was established through interactions with specific residues (Gln789, Thr802 and Met805) and the $\mathrm{Mg}^{2+}$ ion. In addition, residues commonly described in the literature were also computed for these structures: Tyr612, Ser661, Leu725, Phe786, Leu804 and Phe820 [17,27,36]. 


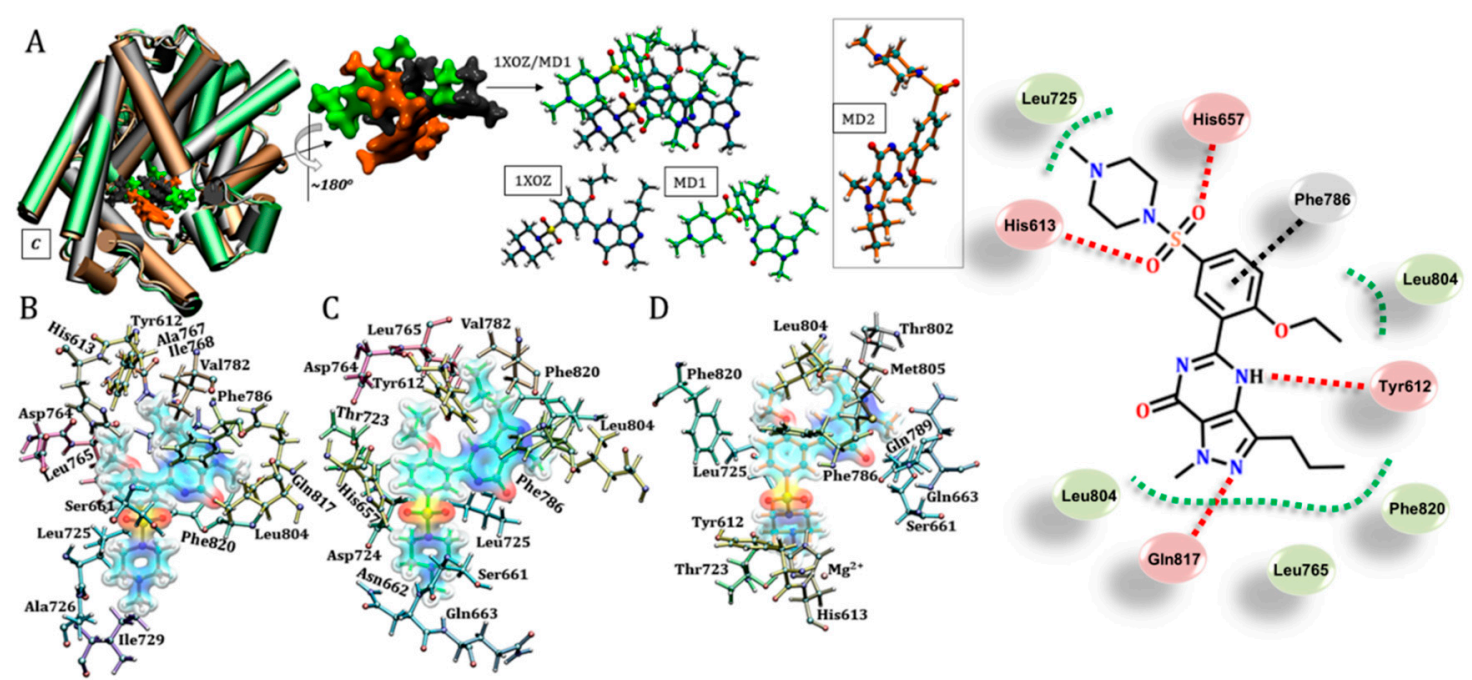

Figure 3. Stabilization of sildenafil by specific amino acid residues in the catalytic pocket, considering the protein structures of $1 \mathrm{XOZ}$ and MD1 from MD simulations. (A) Shows the overlays of the poses considering the different structures, highlighting conformational similarity between 1XOZ and MD1. (B) Residues that stabilize sildenafil considering the crystallographic structure. (C) and (D) show residues interacting with the ligand considering MD1 and MD2, respectively. Residues were $\mathrm{r} \leq 3.0 \AA$ from the ligand. Legend of interactions, red: hydrogen bond (and dipole-dipole); green: hydrophobic; black: $\pi$-stacking.

\subsection{Avanafil (Stendra ${ }^{\circledR} /$ Spedra ${ }^{\circledR}$ )}

Avanafil presented different binding modes according to the protein structures studied here, as shown in Figure 4. In the docking mode for the $1 \mathrm{XOZ}$ structure, the hydroxyl present in the pyrrolidine ring of the inhibitor performed metal-ligand interactions with $\mathrm{Mg}^{2+}$ and Asp764; this aspartate residue was, in turn, stabilized by $\mathrm{Zn}^{2+}$ (see Figure S5 for details). It was interesting to note that the carbonyl from the amide group (located between the pyrimidine rings on avanafil) was able to carry out dipole-dipole interactions, and even form hydrogen bonds: (i) with the hydroxyl from the side chain of Ser661 and (ii) with the amino group of the Gln789 residue. In both situations, the inhibitor acted as the receptor and the protein as the donor in the hydrogen bond. Additionally, Tyr612 utilized its own side chain hydroxyl to make hydrogen bonds with the terminal methoxy of the inhibitor.

There is also the possibility of a $\pi$-stacking type interaction between the aromatic rings of Phe786 and the pyrimidine ring of avanafil. The pyrimidine group can also perform dipole-dipole interactions with Asn662 and His613. Moreover, the imidazole ring from this last residue (His613) can perform electrostatic interactions with the chlorine located in the ligand structure. Finally, a set of nonpolar residues may also stabilize this inhibitor in the catalytic domain of PDE5, specifically Val782, Phe786 and Leu804.

The interactions computed were distinct in each protein structure considered, as shown in Figure 4 (and Figure S5). According to the overall analysis, there were some residues that were able to stabilize both avanafil and tadalafil, such as Tyr612, Val782, Phe786, Leu804, Met816 and Phe820. In addition, $\mathrm{Mg}^{2+}$ and $\mathrm{Zn}^{2+}$ ions were identified close to the avanafil (Figure S5) and similarly observed for sildenafil [27]. Moreover, it is important to highlight the possibility of accommodating the inhibitor avanafil within the catalytic pocket via interactions with other important residues, such as His613, Asp654, His657, Ser661, Asn662, Gln663, Asp724, Leu725, Asp764, Leu765, Ile768, Glu785, Gln789, Thr802, Met805 and Phe820. Interactions with other residues were identified mainly in relation to the structures extracted from the MD simulations, which showed different modes of protein-ligand binding. 

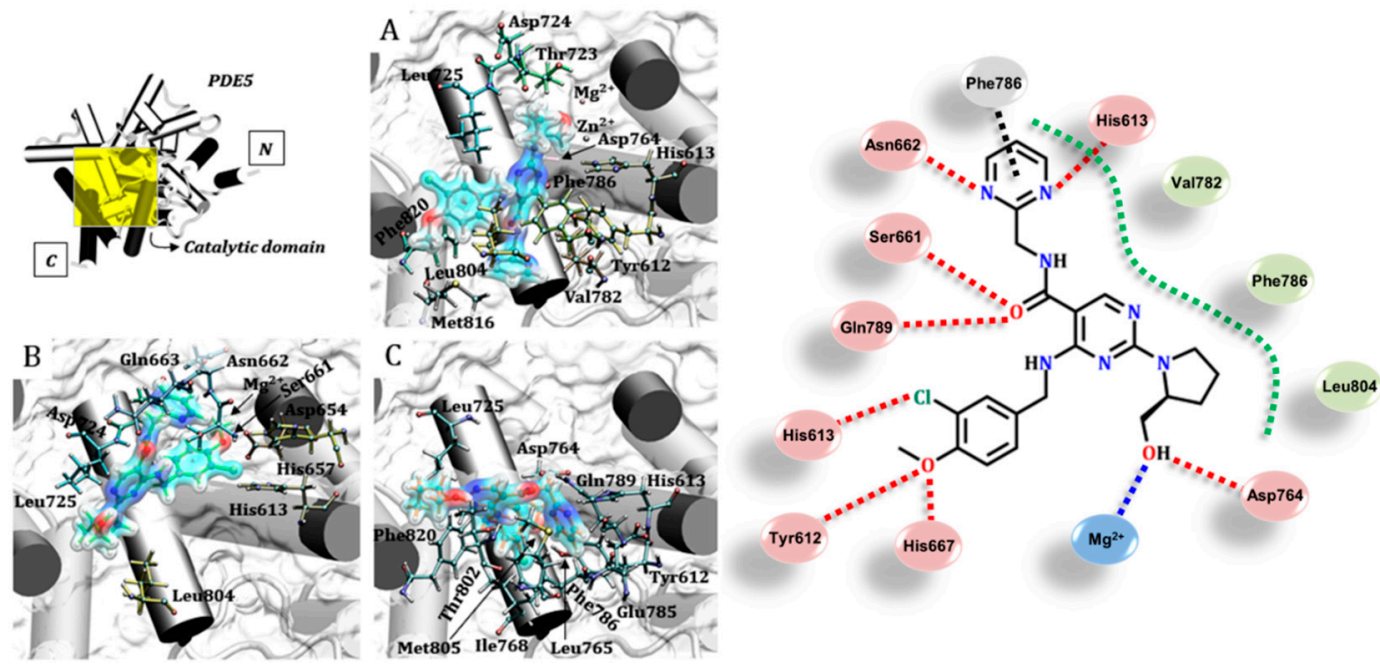

Figure 4. Protein-avanafil interactions in the catalytic site of the PDE5 protein: 1XOZ and the structures from the MD simulations. (A) crystallographic structure; (B) and (C) structures obtained from $80 \mathrm{~ns}$ independent MD simulations, MD1 and MD2, respectively. Residues were $\mathrm{r} \leq 3.0 \AA$ from the ligand. Legend of interactions, red: hydrogen bond (and dipole-dipole); green: hydrophobic; black: $\pi$-stacking; blue: metal-ligand.

There is a lack of information about avanafil stabilization at the molecular level, and the residues highlighted here help to understand, for instance, the pharmacological effects of this ligand [52-55]. Among all PDE5 inhibitors discussed in this study, avanafil is the compound with the least information available in the literature, mainly due to its recent discovery and commercial use [52,53]. Our results shed light on protein-avanafil interactions, detailing the most important amino acid residues for avanafil stabilization, which can contribute to a molecular understanding of the origin of its pharmacological effect $[54,55]$.

\subsection{Udenafil (Zydena $\left.{ }^{\circledR}\right)$}

The udenafil inhibitor showed important interactions from its benzenesulfonamide group. As depicted in Figure 5, for instance, it is possible to observe metal-ligand interactions with the magnesium ion in the crystallographic structure $1 \mathrm{XOZ}$, in addition to the dipole-dipole bond with the imidazole ring of His613 (details in Figure S6). In the structures extracted from MD simulations, there are interactions that suggest hydrogen bonds of the benzenesulfonamide group with the imidazole ring of His657 (MD1) and amine groups of Gln663 and Gln789 (MD2), as well as with the hydroxyl in the side chain of Ser661. According to these interactions, the amino acid residues acted as donors in the hydrogen bond and udenafil was a preferential acceptor. Asn662 also acted as a donor in its hydrogen bonding with the sulfonamide nitrogen of the inhibitor. On the other side of the catalytic site, the Tyr612 residue acted as a receptor when it interacted with the pyrimidine ring of udenafil. Besides polar interactions, hydrophobic and $\pi$-stacking intermolecular bindings were observed between the aromatic rings of Phe820, Phe786 and His613. Although udenafil is the most bulky inhibitor when docked, the set of residues that stabilize it are also observed for the other inhibitors of PDE5, as summarized in Figure 5 [17,27,48]. 


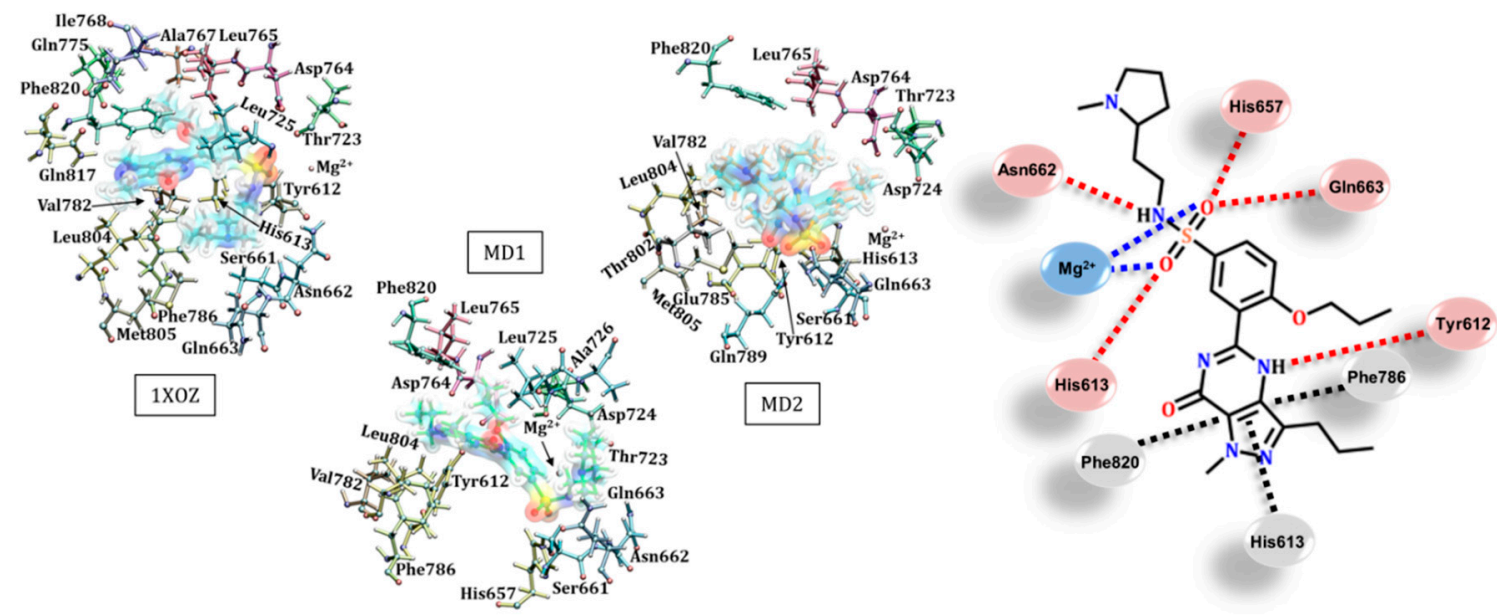

Figure 5. Interactions between protein-udenafil in the catalytic site of PDE5 for crystallographic structure 1XOZ, MD1 and MD2. Different arrangements of the ligand in each structure and some important interactions are highlighted at the right. Residues were $r \leq 3.0 \AA$ from the ligand. Legend of interactions, red: hydrogen bond (and dipole-dipole); black: $\pi$-stacking; blue: metal-ligand.

Udenafil is an efficient inhibitor of PDE5 with rapid absorption, with a maximum plasma concentration occurring between 50 and $80 \mathrm{~min}$ [56]. The $\mathrm{IC}_{50}$ obtained for udenafil ( $5.7 \mathrm{nM}$ ) has an approximate value similar to most traditional inhibitors, such as tadalafil $(5 \mathrm{nM})$ and sildenafil $(6 \mathrm{nM})$, and it is therefore pointed as an interesting alternative for the treatment of erectile dysfunction [57]. Thus, from a molecular point of view, it is relevant to highlight the capacity of the benzenesulfonamide group to form several interactions with different residues in the catalytic site. Considering tadalafil, it is possible to observe that some residues interact with both inhibitors, such as Tyr612, Ala767, Ile768, Gln775, Val782, Phe786, Leu804, Gln817 and Phe820, in addition to mapped residues (His613, Ser661, Asn662, Gln663, Thr723, Leu725, Asp764, Leu765 and Met805) and the magnesium ion. The proximity of the $\mathrm{Mg}^{2+}$ ion in all of the structures used in our molecular docking study is interesting, as it suggests the importance of the $\mathrm{M}$ site in the stabilization of udenafil.

\subsection{Vardenafil (Levitra ${ }^{\circledR}$ )}

Vardenafil has higher inhibitory activity ( $\mathrm{IC}_{50}$ of approximately $0.14-0.70 \mathrm{nM}$ ) than the other compounds studied here ( $\mathrm{IC}_{50}$ around 3.5-9.0 $\mathrm{nM}$ ) [43,57], which motivated us to look for interactions that may help to understand this property. Firstly, a hydrogen bonding interaction with the side chain of Gln817 that was similar to that observed for sildenafil was identified, wherein the ligand acted as the acceptor. This was the converse to the case observed for tadalafil, wherein the ligand acted as the donor. Specifically, the pyrazole ring of sildenafil acted as a binding receptor, a behavior similar to that observed for the imidazole ring of vardenafil. Other protein-ligand interactions observed were: (i) $\pi$-stacking interactions between the benzene rings of Phe786 and Phe 820 with the pyrimidine ring of vardenafil in the crystallographic structure, and (ii) dipole-dipole interactions between the benzenesulfonamide group of the inhibitor with the imidazole ring of His657 (structure MD1) and the hydroxyl of Ser661, as well as the amino group of the side chain of Gln789 (MD2). These interactions are detailed in Figure 6. Two important interactions from the pyrimidine ring of this inhibitor suggest possible hydrogen bonds with Tyr612 (MD1) and His613 (MD2), with these residues acting as acceptor and donor, respectively, as shown Figure S7. It is important to note that one of the structures extracted from the MD simulations was able to capture the residue Tyr612, which contributes to the high affinity of vardenafil compared to sildenafil, as previously proposed [58]. Finally, some hydrophobic contributions were also observed between the inhibitor and residues of Leu765, Val782, Leu804 and Phe820. 


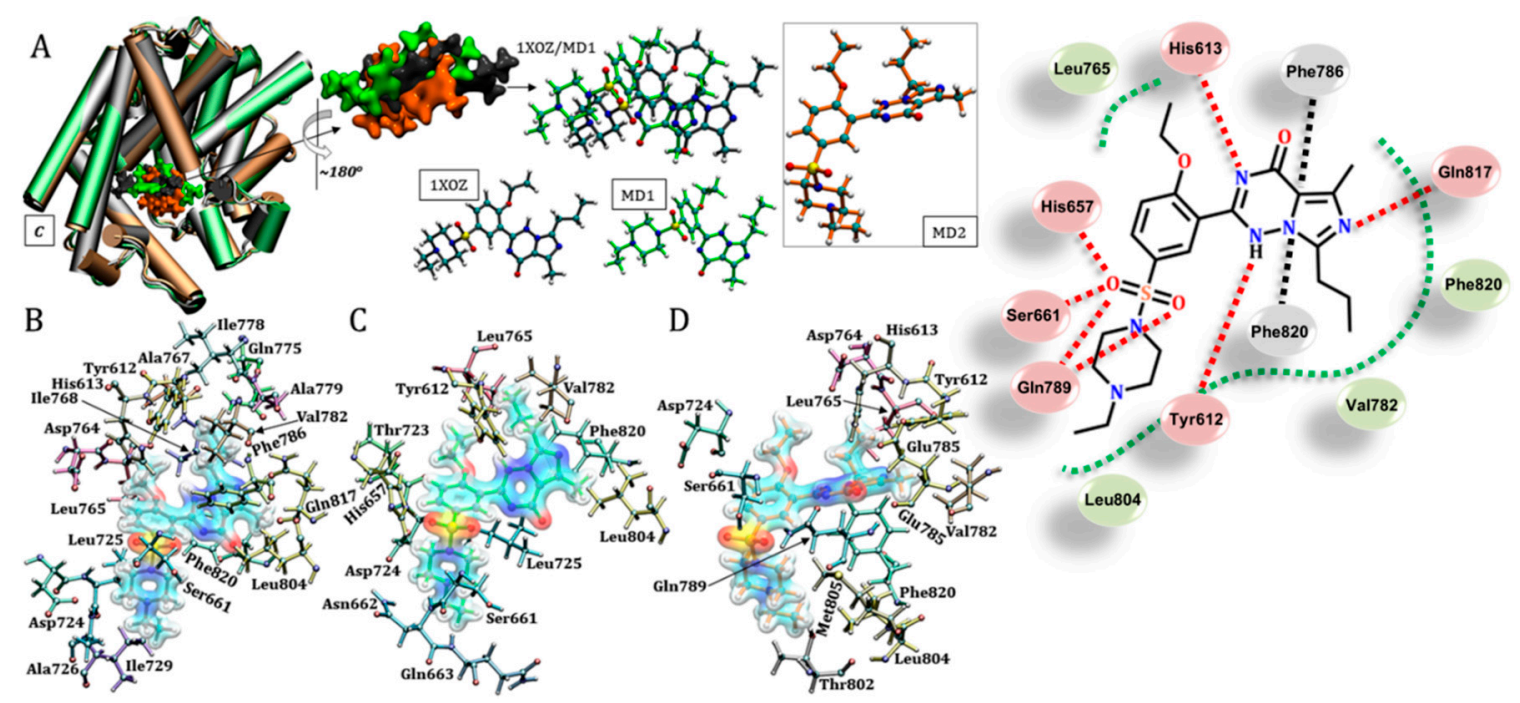

Figure 6. Positions of the vardenafil ligand obtained for the three protein structures studied. (A) shows the position similarity obtained for vardenafil in the 1XOZ and MD1 structures. (B) Shows the residues in the crystallographic structure that stabilize the ligand, while $(\mathbf{C})$ and $(\mathbf{D})$ show the residues responsible for inhibitor stabilization in the catalytic site considering structures MD1 and MD2, respectively. Residues were $r \leq 3.0 \AA$ from the ligand. Legend of interactions, red: hydrogen bond (and dipole-dipole); green: hydrophobic; black: $\pi$-stacking.

As a commercial drug, vardenafil is one of the most potent inhibitors of phosphodiesterase type 5 available for clinical treatment. Curiously, despite its structural similarity to sildenafil, this inhibitor has an $\mathrm{IC}_{50}$ only $2.3 \%$ of the sildenafil concentration required for the same PDE5 inhibitory effect. The binding mode in the catalytic pocket is similar for both compounds and a high efficiency can be suggested considering the bioavailability process and/or a contribution of Leu804 for terminal methyl amine stabilization $[57,59]$. Thus, many residues stabilize both sildenafil and vardenafil at the catalytic site, such as Tyr612, His613, Ser661, Asn662, Thr723, Asp724, Leu725, Ala726, Ile729, Asp764, Leu765, Al767, Ile768, Val782, Phe786, Leu804, Gln817 and Phe820; in addition to His657, Gln663, Gln775, Ile778 and Ala779, considering the crystallographic and MD1 structures. On the other hand, considering the MD2 structure from the MD simulation, the conformation assumed by the inhibitor vardenafil (and sildenafil, see Figure 3) was significantly different and the identified residues that stabilized vardenafil were Tyr612, His613, Ser661, Asp724, Leu765, Val782, Glu785, Gln789, Thr802, Leu804, Met805 and Phe820.

\subsection{General Mapping of PDE5-Ligands Interactions in the Catalytic Domain}

The commercial drugs available for phosphodiesterase type 5 inhibition have singularities in their structures which affect their stabilization via several residues in the catalytic domain. The contribution of PDE5-ligand interactions is reflected in the experimental results of inhibitory activity, for instance $\mathrm{IC}_{50}$ : avanafil $1 \mathrm{nM}$; sildenafil $3.7 \mathrm{nM}$; tadalafil $1.8 \mathrm{nM}$; udenafil $8.2 \mathrm{nM}$ and vardenafil $0.09 \mathrm{nM}$ [60]. Despite vardenafil having the highest potency among the other drugs studied here, the selectivity of this compound still requires study. Adverse effects are also directly related to the potency of vardenafil and, due to its action in other phosphodiesterases, are a concern [61]. Considering only the action at the PDE5 catalytic domain, a general mechanism for PDE5 inhibition suggests an interaction which avoids the hydrolysis of cGMP to GMP, and there are specific residues essential for the binding of cGMP, as well as inhibitors [27]. The results discussed here present a pattern of interactions within the catalytic pocket of PDE5, with some residues being highly versatile, assuming different conformations and interactions to better stabilize the ligands in this region. The frequency of observation of these residues is presented as probabilities in Figure 7, which shows the importance of each component in 
the docking of the commercial drugs evaluated. Residues such as Tyr612, Leu725, Leu804 and Phe820 were frequently found close to the ligands $(r \leq 3.0 \AA)$. In fact, Tyr612 has been described in the literature as important for increasing the affinity of vardenafil compared to tadalafil [58], as indicated in the present study. Other residues also showed a high probability of being found in "contact" with the PDE5 inhibitors, including Phe786, Val782, Leu765, Asp764, Asp724, Thr723, Ser661 and His613, as well as the magnesium ion. It is important to highlight that amino acid residues with hydrophobic side chains do not necessarily form only non-polar interactions with the inhibitors; they can use the backbone carbonyl (or amine) to form polar interactions, even with well-directed hydrogen bonds [62] (according the probabilities depicted in Figure 7).
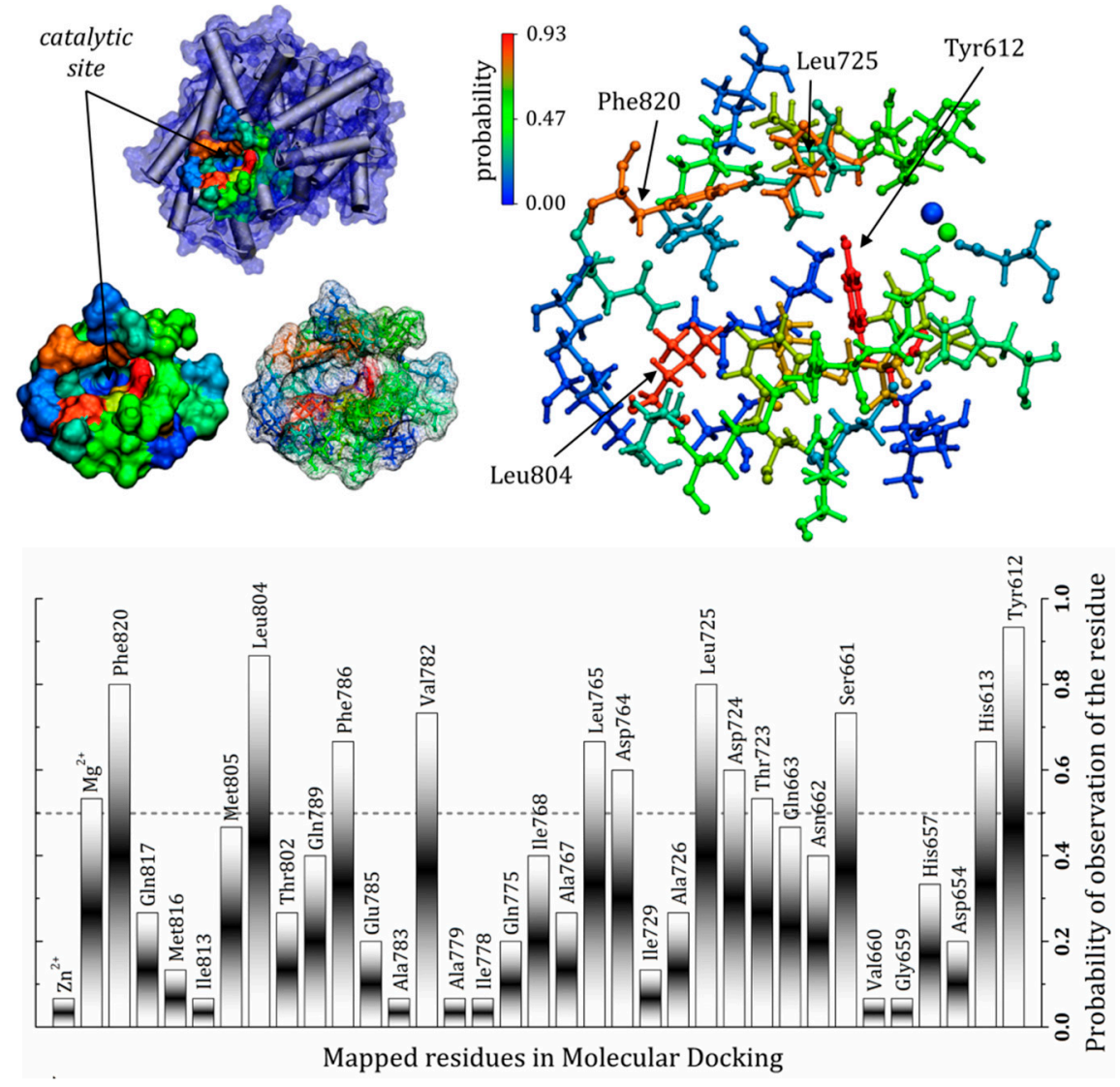

Figure 7. Amino acid residues identified as important for stabilization of the commercial PDE5 inhibitors, showing the frequency of observation of the residues as a probability. The probability values were obtained by the number of observations considering all the inhibitors and the three structures used in the molecular docking study. Residues were $r \leq 3.0$ A from the ligand.

\section{Conclusions}

The in silico results discussed here describe in detail the interactions between the five main commercially available PDE5 inhibitors (tadalafil, sildenafil, avanafil, udenafil and vardenafil) and the residues that make up the catalytic domain of the PDE5 enzyme. The structures extracted from MD simulations allowed for observation of new interactions by PDE5 inhibitors, suggesting a set of possibilities for contact that has not yet been described in the literature. This was possible because the correlation with the initial crystallographic structure was decreased. Docking of the inhibitors using a minimized structure in aqueous solvent (MD1 and MD2) allowed us to observe new interactions and, as a consequence, a set of essential residues was identified and presented as a probabilities value, where the most likely residues were Tyr612, Phe786, Val782, Leu765, Asp764, Asp724, Thr723, Ser661 and His613. 
Supplementary Materials: The following are available online at http://www.mdpi.com/2218-0532/87/4/29/s1.

Author Contributions: Conceptualization, I.P.d.O., C.H.L. and G.D.N.; methodology, validation and formal analysis, I.P.d.O.; writing, review and editing, I.P.d.O. and C.H.L.; supervision and funding acquisition, G.D.N.

Funding: The authors would like to thank the financial support of FAPESP (C.H.L. postdoc fellowship FAPESP process number 2017/26687-3 and I.P.O. postdoc fellowship FAPESP process number 2017/02201-4). CNPq grant 470374/2013-6 and FAPESP (grants 349 2010/16947-9, 2013/05475-7, 2013/08293-7, 2013/22360-9).

Conflicts of Interest: The authors declare no conflict of interest.

\section{References}

1. Francis, S.H.; Turko, I.V.; Corbin, J.D. Cyclic nucleotide phosphodiesterases: Relating structure and function. Prog. Nucleic Acid Res. Mol. Biol. 2001, 65, 1-52. [PubMed]

2. Corbin, J.D.; Francis, S.H.; Webb, D.J. Phosphodiesterase type 5 as a pharmacologic target in erectile dysfunction. Urology 2002, 60, 4-11. [CrossRef]

3. Soderling, S.H.; Beavo, J.A. Regulation of cAMP and cGMP signaling: New phosphodiesterases and new functions. Curr. Opin. Cell Biol. 2000, 12, 174-179. [CrossRef]

4. Tsai, E.J.; Kass, D.A. Cyclic GMP signaling in cardiovascular pathophysiology and therapeutics. Pharmacol. Ther. 2009, 122, 216-238. [CrossRef]

5. Arshad, N.; Visweswariah, S.S. The multiple and enigmatic roles of guanylyl cyclase C in intestinal homeostasis. FEBS Lett. 2012, 586, 2835-2840. [CrossRef]

6. Mendes-Silverio, C.B.; Leiria, L.O.S.; Morganti, R.P.; Anhê, G.F.; Marcondes, S.; Mónica, F.Z.; De Nucci, G.; Antunes, E. Activation of Haem-Oxidized Soluble Guanylyl Cyclase with BAY 60-2770 in Human Platelets Lead to Overstimulation of the Cyclic GMP Signaling Pathway. PLoS ONE 2012, 7, e47223. [CrossRef]

7. Sinðić, A.; Hirsch, J.R.; Velic, A.; Piechota, H.; Schlatter, E.; Sinic, A.; Sin, J.R.H.A. Guanylin and uroguanylin regulate electrolyte transport in isolated human cortical collecting ducts. Kidney Int. 2005, 67, 1420-1427. [CrossRef]

8. Willipinski-Stapelfeldt, B.; Lübberstedt, J.; Stelter, S.; Vogt, K.; Mukhopadhyay, A.K.; Müller, D. Comparative analysis between cyclic GMP and cyclic AMP signalling in human sperm. Mol. Hum. Reprod. 2004, 10, 543-552. [CrossRef]

9. Bian, K.; Murad, F. sGC-cGMP Signaling: Target for Anticancer Therapy. Adv. Exp. Medicine Biol. 2014, 814, 5-13.

10. Francis, S.H.; Busch, J.L.; Corbin, J.D. cGMP-dependent protein kinases and cGMP phosphodiesterases in nitric oxide and cGMP action. Pharmacol. Rev. 2010, 62, 525-563. [CrossRef]

11. Murad, F. Nitric Oxide and Cyclic GMP in Cell Signaling and Drug Development. N. Engl. J. Med. 2006, 355, 2003-2011. [CrossRef] [PubMed]

12. Beavo, J.A. Cyclic nucleotide phosphodiesterases: Functional implications of multiple isoforms. Physiol. Rev. 1995, 75, 725-748. [CrossRef] [PubMed]

13. Morelli, A.; Filippi, S.; Mancina, R.; Luconi, M.; Vignozzi, L.; Marini, M.; Orlando, C.; Vannelli, G.B.; Aversa, A.; Natali, A.; et al. Androgens Regulate Phosphodiesterase Type 5 Expression and Functional Activity in Corpora Cavernosa. Endocrinology 2004, 145, 2253-2263. [CrossRef] [PubMed]

14. Li, Z.; Xi, X.; Gu, M.; Feil, R.; Ye, R.D.; Eigenthaler, M.; Hofmann, F.; Du, X. A stimulatory role for cGMP-dependent protein kinase in platelet activation. Cell 2003, 112, 77-86. [CrossRef]

15. Wallis, R.M.; Corbin, J.D.; Francis, S.H.; Ellis, P. Tissue distribution of phosphodiesterase families and the effects of sildenafil on tissue cyclic nucleotides, platelet function, and the contractile responses of trabeculae carneae and aortic rings in vitro. Am. J. Cardiol. 1999, 83, 3-12. [CrossRef]

16. Mullershausen, F.; Russwurm, M.; Thompson, W.J.; Liu, L.; Koesling, D.; Friebe, A. Rapid nitric oxide-induced desensitization of the cGMP response is caused by increased activity of phosphodiesterase type 5 paralleled by phosphorylation of the enzyme. J. Cell Boil. 2001, 155, 271-278. [CrossRef] [PubMed]

17. Zoraghi, R.; Francis, S.H.; Corbin, J.D. Critical Amino Acids in Phosphodiesterase-5 Catalytic Site That Provide for High-Affinity Interaction with Cyclic Guanosine Monophosphate and Inhibitors. Biochemistry 2007, 46, 13554-13563. [CrossRef]

18. Boolell, M.; Gepi-Attee, S.; Gingell, J.; Allen, M. Sildenafil, a novel effective oral therapy for male erectile dysfunction. BJU Int. 1996, 78, 257-261. [CrossRef] 
19. Rotella, D.P. Phosphodiesterase 5 inhibitors: Current status and potential applications. Nat. Rev. Drug Discov. 2002, 1, 674-682. [CrossRef]

20. Haning, H.; Niewöhner, U.; Bischoff, E. Phosphodiesterase type 5 (PDE5) inhibitors. Prog. Med. Chem. 2003, 41, 249-306.

21. Mergia, E.; Stegbauer, J. Role of Phosphodiesterase 5 and Cyclic GMP in Hypertension. Curr. Hypertens. Rep. 2016, 18, 39. [CrossRef] [PubMed]

22. Archer, S.L.; Michelakis, E.D. Phosphodiesterase Type 5 Inhibitors for Pulmonary Arterial Hypertension. N. Engl. J. Med. 2009, 361, 1864-1871. [CrossRef] [PubMed]

23. Tinel, H.; Hütter, J.; Sandner, P.; Stelte-Ludwig, B.; Stelte-Ludwig, B. Pre-clinical evidence for the use of phosphodiesterase- 5 inhibitors for treating benign prostatic hyperplasia and lower urinary tract symptoms. BJU Int. 2006, 98, 1259-1263. [CrossRef] [PubMed]

24. Corbin, J.D.; Beasley, A.; Blount, M.A.; Francis, S.H. High lung PDE5: A strong basis for treating pulmonary hypertension with PDE5 inhibitors. Biochem. Biophys. Res. Commun. 2005, 334, 930-938. [CrossRef]

25. Barone, I.; Giordano, C.; Bonofiglio, D.; Catalano, S.; Andò, S. Phosphodiesterase Type 5 as a Candidate Therapeutic Target in Cancers. Curr. Pathobiol. Rep. 2015, 3, 193-201. [CrossRef]

26. Mónica, F.Z.; De Nucci, G. Tadalafil for the treatment of benign prostatic hyperplasia. Expert Opin. Pharmacother. 2019, 1-9. [CrossRef]

27. Sung, B.-J.; Hwang, K.Y.; Jeon, Y.H.; Lee, J.I.; Heo, Y.-S.; Kim, J.H.; Moon, J.; Yoon, J.M.; Hyun, Y.-L.; Kim, E.; et al. Structure of the catalytic domain of human phosphodiesterase 5 with bound drug molecules. Nature 2003, 425, 98-102. [CrossRef]

28. Zenzmaier, C.; Sampson, N.; Pernkopf, D.; Plas, E.; Untergasser, G.; Berger, P. Attenuated Proliferation and Trans -Differentiation of Prostatic Stromal Cells Indicate Suitability of Phosphodiesterase Type 5 Inhibitors for Prevention and Treatment of Benign Prostatic Hyperplasia. Endocrinology 2010, 151, 3975-3984. [CrossRef]

29. Catalano, S.; Campana, A.; Giordano, C.; Győrffy, B.; Tarallo, R.; Rinaldi, A.; Bruno, G.; Ferraro, A.; Romeo, F.; Lanzino, M.; et al. Expression and function of phosphodiesterase type 5 in human breast cancer cell lines and tissues: Implications for targeted therapy. Clin. Cancer Res. 2016, 22, 2271-2282. [CrossRef]

30. Cahill, K.B.; Quade, J.H.; Carleton, K.L.; Cote, R.H. Identification of Amino Acid Residues Responsible for the Selectivity of Tadalafil Binding to Two Closely Related Phosphodiesterases, PDE5 and PDE6*. J. Boil. Chem. 2012, 287, 41406-41416. [CrossRef]

31. Kayık, G.; Tüzün, N.Ş.; Durdagi, S. Investigation of PDE5/PDE6 and PDE5/PDE11 selective potent tadalafil-like PDE5 inhibitors using combination of molecular modeling approaches, molecular fingerprint-based virtual screening protocols and structure-based pharmacophore development. J. Enzym. Inhib. Med. Chem. 2017, 32, 311-330. [CrossRef] [PubMed]

32. Pattis, J.G.; Kamal, S.; Li, B.; May, E.R. Catalytic Domains of Phosphodiesterase 5, 6, and 5/6 Chimera Display Differential Dynamics and Ligand Dissociation Energy Barriers. J. Phys. Chem. B 2019, 123, 825-835. [CrossRef] [PubMed]

33. Chan-Tack, K.M. Oral sildenafil in erectile dysfunction. Lancet 1998, 352, 1557. [CrossRef]

34. Lugnier, C. Cyclic nucleotide phosphodiesterase (PDE) superfamily: A new target for the development of specific therapeutic agents. Pharmacol. Ther. 2006, 109, 366-398. [CrossRef]

35. Zoraghi, R.; Bessay, E.P.; Corbin, J.D.; Francis, S.H. Structural and Functional Features in Human PDE5A1 Regulatory Domain That Provide for Allosteric cGMP Binding, Dimerization, and Regulation. J. Boil. Chem. 2005, 280, 12051-12063. [CrossRef]

36. Card, G.L.; England, B.P.; Suzuki, Y.; Fong, D.; Powell, B.; Lee, B.; Luu, C.; Tabrizizad, M.; Gillette, S.; Ibrahim, P.N.; et al. Structural Basis for the Activity of Drugs that Inhibit Phosphodiesterases. Structure 2004, 12, 2233-2247. [CrossRef]

37. Martinez, L.; Andrade, R.; Birgin, E.G.; Martínez, J.M. PACKMOL: A package for building initial configurations for molecular dynamics simulations. J. Comput. Chem. 2009, 30, 2157-2164. [CrossRef]

38. Chandrasekhar, J.; Impey, R.W.; Jorgensen, W.L.; Madura, J.D.; Klein, M.L. Comparison of simple potential functions for simulating liquid water. J. Chem. Phys. 1983, 79, 926.

39. Phillips, J.C.; Braun, R.; Wang, W.; Gumbart, J.; Tajkhorshid, E.; Villa, E.; Chipot, C.; Skeel, R.D.; Kale, L.; Schulten, K. Scalable molecular dynamics with NAMD. J. Comput. Chem. 2005, 26, 1781-1802. [CrossRef]

40. Humphrey, W.; Dalke, A.; Schulten, K. VMD: Visual molecular dynamics. J. Mol. Graph. 1996, 14, 33-38. [CrossRef] 
41. Xie, X.-Q.S. Exploiting PubChem for virtual screening. Expert Opin. Drug Discov. 2010, 5, 1205-1220. [CrossRef] [PubMed]

42. de Magalhães, C.S.; Almeida, D.M.; Barbosa, H.J.C.; Dardenne, L.E. A dynamic niching genetic algorithm strategy for docking highly flexible ligands. Inf. Sci. (N. Y.) 2014, 289, 206-224. [CrossRef]

43. Seftel, A.D. Phosphodiesterase type 5 inhibitors: Molecular pharmacology and interactions with other phosphodiesterases. Curr. Pharm. Des. 2005, 11, 4047-4058. [CrossRef]

44. Erickson, J.A.; Jalaie, M.; Robertson, D.H.; Lewis, R.A.; Vieth, M. Lessons in Molecular Recognition: The Effects of Ligand and Protein Flexibility on Molecular Docking Accuracy. J. Med. Chem. 2004, 47, 45-55. [CrossRef]

45. Spyrakis, F.; BidonChanal, A.; Barril, X.; Luque, F.J. Protein flexibility and ligand recognition: Challenges for molecular modeling. Curr. Top. Med. Chem. 2011,11, 192-210. [CrossRef]

46. Huang, S.Y.; Zou, X. Ensemble docking of multiple protein structures: Considering protein structural variations in molecular docking. Proteins Struct. Funct. Genet. 2007, 66, 399-421. [CrossRef]

47. Osterberg, F.; Morris, G.M.; Sanner, M.F.; Olson, A.J.; Goodsell, D.S. Automated docking to multiple target structures: Incorporation of protein mobility and structural water heterogeneity in AutoDock. Proteins Struct. Funct. Bioinform. 2002, 46, 34-40. [CrossRef]

48. Zoraghi, R.; Corbin, J.D.; Francis, S.H. Phosphodiesterase-5 Gln817 is critical for cGMP, vardenafil, or sildenafil affinity: Its orientation impacts cGMP but not cAMP affinity. J. Biol. Chem. 2006, 281, 5553-5558. [CrossRef]

49. de Oliveira, I.P.; Lescano, C.H.; De Nucci, G. Q817G mutation in phosphodiesterase type 5 (PDE-5): Conformational analysis and dissociation profile of the inhibitor Tadalafil. Chem. Biol. Drug Des. 2018, 93, 419-429. [CrossRef]

50. Dill, K.A.; Maccallum, J.L. The Protein-Folding Problem, 50 Years On. Science 2012, 338, 1042-1046. [CrossRef]

51. Wang, H.; Liu, Y.; Huai, Q.; Cai, J.; Zoraghi, R.; Francis, S.H.; Corbin, J.D.; Robinson, H.; Xin, Z.; Lin, G.; et al. Multiple conformations of phosphodiesterase-5: Implications for enzyme function and drug development. J. Biol. Chem. 2006, 281, 21469-21479. [CrossRef] [PubMed]

52. Masche, U.P. Avanafil. Pharma Krit. 2016, 38, 13-14. [CrossRef]

53. Sanford, M. Avanafil: A Review of Its Use in Patients with Erectile Dysfunction. Drugs Aging 2013, 30, 853-862. [CrossRef] [PubMed]

54. Burke, R.M.; Evans, J.D. Avanafil for treatment of erectile dysfunction: Review of its potential. Vasc. Health Risk Manag. 2012, 8, 517-523.

55. Kotera, J.; Mochida, H.; Inoue, H.; Noto, T.; Fujishige, K.; Sasaki, T.; Kobayashi, T.; Kojima, K.; Yee, S.; Yamada, Y.; et al. Avanafil, a Potent and Highly Selective Phosphodiesterase-5 Inhibitor for Erectile Dysfunction. J. Urol. 2012, 188, 668-674. [CrossRef] [PubMed]

56. Kang, S.G.; Kim, J.J. Udenafil: Efficacy and tolerability in the management of erectile dysfunction. Ther. Adv. Urol. 2013, 5, 101-110. [CrossRef] [PubMed]

57. Huang, Y.-Y.; Li, Z.; Cai, Y.-H.; Feng, L.-J.; Wu, Y.; Li, X.; Luo, H.-B. The Molecular Basis for the Selectivity of Tadalafil toward Phosphodiesterase 5 and 6: A Modeling Study. J. Chem. Inf. Model. 2013, 53, 3044-3053. [CrossRef]

58. Corbin, J.; Francis, S.; Zoraghi, R. Tyrosine-612 in PDE5 contributes to higher affinity for vardenafil over sildenafil. Int. J. Impot. Res. 2006, 18, 251-257. [CrossRef]

59. Hellstrom, W.J.G. Vardenafil: A new approach to the treatment of erectile dysfunction. Curr. Urol. Rep. 2003, 4, 479-487. [CrossRef]

60. Palit, V.; Eardley, I. An update on new oral PDE5 inhibitors for the treatment of erectile dysfunction. Nat. Rev. Urol. 2010, 7, 603-609. [CrossRef]

61. Corbin, J.D.; Rannels, S.R.; Francis, S.H. Phosphodiesterase-5 Inhibition BT-Heart Disease and Erectile Dysfunction; Kloner, R.A., Ed.; Humana Press: Totowa, NJ, USA, 2004; pp. 117-130. [CrossRef]

62. Oliveira, I.P.; Martínez, L. Molecular basis for competitive solvation of the Burkholderia cepacia lipase by sorbitol and urea. Phys. Chem. Chem. Phys. 2016, 18, 21797-21808. [CrossRef] [PubMed]

(C) 2019 by the authors. Licensee MDPI, Basel, Switzerland. This article is an open access article distributed under the terms and conditions of the Creative Commons Attribution (CC BY) license (http://creativecommons.org/licenses/by/4.0/). 